\title{
Some Approximation Properties of $q$-Baskakov-Beta-Stancu Type Operators
}

\author{
Vishnu Narayan Mishra, ${ }^{1}$ Kejal Khatri, ${ }^{1}$ and Lakshmi Narayan Mishra ${ }^{2,3}$ \\ ${ }^{1}$ Department of Applied Mathematics and Humanities, Sardar Vallabhbhai National Institute of Technology, \\ Ichchhanath Mahadev Road, Surat, Gujarat 395 007, India \\ ${ }^{2}$ L. 1627 Awadhpuri Colony Beniganj, Opp. I.T.I. Ayodhya Main Road, Faizabad, Uttar Pradesh 224 001, India \\ ${ }^{3}$ Department of Mathematics, National Institute of Technology, Silchar, Cachar District, Assam 788 010, India
}

Correspondence should be addressed to Vishnu Narayan Mishra; vishnunarayanmishra@gmail.com

Received 7 May 2013; Accepted 8 October 2013

Academic Editor: Adam Kowalewski

Copyright (C) 2013 Vishnu Narayan Mishra et al. This is an open access article distributed under the Creative Commons Attribution License, which permits unrestricted use, distribution, and reproduction in any medium, provided the original work is properly cited.

This paper deals with new type $q$-Baskakov-Beta-Stancu operators defined in the paper. First, we have used the properties of $q$ integral to establish the moments of these operators. We also obtain some approximation properties and asymptotic formulae for these operators. In the end we have also presented better error estimations for the $q$-operators.

\section{Introduction}

In the recent years, the quantum calculus ( $q$-calculus) has attracted a great deal of interest because of its potential applications in mathematics, mechanics, and physics. Due to the applications of $q$-calculus in the area of approximation theory, q-generalization of some positive operators has attracted much interest, and a great number of interesting results related to these operators have been obtained (see, for instance, [1-3]). In this direction, several authors have proposed the $q$-analogues of different linear positive operators and studied their approximation behaviors. Also, Aral and Gupta [4] defined q-generalization of the Baskakov operators and investigated some approximation properties of these operators. Subsequently, Finta and Gupta [5] obtained global direct error estimates for these operators using the second-order Ditzian Totik modulus of smoothness. To approximate Lebesgue integrable functions on the interval $[0, \infty)$, modified Beta operators $[6]$ are defined as

$$
B_{n}(f, x)=\frac{n-1}{n} \sum_{k=0}^{\infty} b_{n, k}(x) \int_{0}^{\infty} p_{n, k}(t) f(t) d t, \quad x \in[0, \infty),
$$

where $b_{n, k}(x)=(1 / B(k+1, n))\left(x^{k} /(1+x)^{n+k+1}\right)$ and $p_{n, k}(t)=$ $\left(\begin{array}{c}n+k+1 \\ k\end{array}\right)\left(t^{k} /(1+t)^{n+k}\right)$.

The discrete $q$-Beta operators are defined as

$$
V_{n}^{q}(f, x)=\frac{1}{[n]_{q}} \sum_{k=0}^{\infty} b_{n, k}^{q}(x) f\left(\frac{[k]_{q}}{[n+1]_{q} q^{k-1}}\right) .
$$

Recently, Maheshwari and Sharma [7] introduced the $q$ analogue of the Baskakov-Beta-Stancu operators and studied the rate of approximation and weighted approximation of these operators. Motivated by the Stancu type generalization of $q$-Baskakov operators, we propose the $q$-analogue of the operators $B_{n}^{(\alpha, \beta)}$, recently introduced and studied for special values $\alpha=\beta=0$ by Gupta and Kim [8] as

$$
\begin{aligned}
B_{n, \alpha, \beta}^{q}(f, x):= & \frac{[n-1]_{q}}{[n]_{q}} \sum_{k=0}^{\infty} b_{n, k}^{q}(x) \\
& \times \int_{0}^{\infty / A} q^{k} p_{n, k}^{q}(t) f\left(\frac{[n]_{q} t+\alpha}{[n]_{q}+\beta}\right) d_{q} t,
\end{aligned}
$$

where $b_{n, k}^{q}(x)=\left(q^{k(k-1) / 2} / B_{q}(k+1, n)\right)\left(x^{k} /(1+x)_{q}^{n+k+1}\right)$ and $p_{n, k}^{q}(t)=\left(\begin{array}{c}n+k-1 \\ k\end{array}\right)\left(q_{q}^{k(k-1) / 2} / B_{q}(k+1, n)\right)\left(t^{k} /(1+t)_{q}^{n+k+1}\right)$. 
We know that $\sum_{k=0}^{\infty} b_{n, k}^{q}(x)=[n]_{q}$ and $\int_{0}^{\infty / A} q^{k} p_{n, k}^{q}(t)=$ $1 /[n-1]_{q}$. We mention that $B_{n, 0,0}^{q} \equiv B_{n}^{q}$ (see [8]).

Very recently, Gupta et al. [9] introduced some direct results in simultaneous approximation for BaskakovDurrmeyer-Stancu operators. The aim of this paper is to study the approximation properties of a new generalization of the Baskakov type Beta Stancu operators based on qintegers. We estimate moments for these operators. Also, we study asymptotic formula for these operators. Finally, we give better error estimations for the operator (3). First, we recall some definitions and notations of $q$-calculus. Such notations can be found in $[10,11]$. We consider $q$ as a real number satisfying $q>0$. For $n \in \mathbb{N}$,

$$
\begin{gathered}
{[n]_{q}:= \begin{cases}\frac{1-q^{n}}{1-q}, & q \neq 1, \\
n, & q=1,\end{cases} } \\
{[n]_{q} !:= \begin{cases}{[n]_{q}[n-1]_{q}[n-2]_{q}, \ldots,[1]_{q},} & n=1,2, \ldots, \\
1, & n=0,\end{cases} } \\
(1+x)_{q}^{n}:=\prod_{j=0}^{n-1}\left(1+q^{j} x\right) .
\end{gathered}
$$

The $q$-binomial coefficients are given by

$$
\left(\begin{array}{l}
n \\
k
\end{array}\right)_{q}=\frac{[n]_{q} !}{[k]_{q} ![n-k]_{q} !}, \quad 0 \leq k \leq n .
$$

The $q$-derivative $\mathscr{D}_{q} f$ of a function $f$ is given by

$$
\left(\mathscr{D}_{q} f\right)(x)=\frac{f(x)-f(q x)}{(1-q) x}, \quad \text { if } x \neq 0 .
$$

The $q$-analogues of product and quotient rules are defined as

$$
\begin{gathered}
\mathscr{D}_{q}(f(x) g(x))=g(x) \mathscr{D}_{q} f(x)+f(q x) \mathscr{D}_{q} g(x), \\
\mathscr{D}_{q}\left(\frac{f(x)}{g(x)}\right)=\frac{g(x) \mathscr{D}_{q} f(x)-f(x) \mathscr{D}_{q} g(x)}{g(x) g(q x)} .
\end{gathered}
$$

The $q$-Jackson integrals and the $q$-improper integrals are defined as $[12,13]$

$$
\begin{gathered}
\int_{0}^{a} f(x) d_{q} x=(1-q) a \sum_{n=0}^{\infty} f\left(a q^{n}\right) q^{n}, \quad a>0, \\
\int_{0}^{\infty / A} f(x) d_{q} x=(1-q) \sum_{n=-\infty}^{\infty} f\left(\frac{q^{n}}{A}\right) \frac{q^{n}}{A}, \quad A>0,
\end{gathered}
$$

provided that the sums converge absolutely. Using (9), De Sole and Kac [14] defined the $q$-analogue of Beta functions of second kind $B(t, s)=\int_{0}^{\infty}\left(x^{t-1} /(1+x)^{t+s}\right) d x$ as follows:

$$
B_{q}(t, s)=K(A, t) \int_{0}^{\infty / A} \frac{x^{t-1}}{(1+x)_{q}^{t+s}} d_{q} x,
$$

where $K(x, t)=(1 /(1+x)) x^{t}(1+1 / x)_{q}^{t}(1+x)_{q}^{1-t}$. This function is $q$-constant in $x$; that is, $K(q x, t)=K(x, t)$. It was observed in [14] that $B_{q}(t, s)$ is independent of $A$; this is because from the integral and $K(A, t)$ the term $A$ cancels out. In particular for any positive integer $n$, we have

$$
K(x, n)=q^{n(n-1) / 2}, \quad K(x, 0)=1 .
$$

Also, we have

$$
B_{q}(t, s)=\frac{[t-1]_{q} ![s-1]_{q} !}{[t+s-1]_{q} !} .
$$

In [8], Gupta and Kim obtained recurrence formula for the moments of the operators as follows.

Theorem 1 (see [8]). If one defined the central moments as

$$
\begin{aligned}
T_{n, m}(x):= & B_{n}^{q}\left(t^{m} ; x\right)=\frac{[n-1]_{q}}{[n]_{q}} \sum_{k=0}^{\infty} b_{n, k}^{q}(x) \\
& \times \int_{0}^{\infty / A} q^{k} p_{n, k}^{q}(t) t^{m} d_{q} t,
\end{aligned}
$$

then, for $n>m+2$, one has the following recurrence relation:

$$
\begin{aligned}
\left([n]_{q}-\right. & {\left.[m+2]_{q}\right) T_{n, m+1}(q x) } \\
= & q x(1+x) D_{q}\left[T_{n, m}(x)\right] \\
& +q\left([m+1]_{q}+[n+1]_{q} x\right) T_{n, m}(q x) .
\end{aligned}
$$

\section{Moment Estimates}

Lemma 2 (see [8]). The following equalities hold.

(i) $B_{n}^{q}(1, x)=1$.

(ii) $B_{n}^{q}(t, x)=\left([n+1]_{q} / q^{2}[n-2]_{q}\right) x+\left(1 / q[n-2]_{q}\right)$.

(iii) $B_{n}^{q}\left(t^{2}, x\right)=\left([n+1]_{q}[n+2]_{q} / q^{6}[n-2]_{q}[n-3]_{q}\right) x^{2}+([n+$ $\left.1]_{q}[2]_{q}^{2} / q^{5}[n-2]_{q}[n-3]_{q}\right) x+\left([2]_{q} / q^{3}[n-2]_{q}[n-3]_{q}\right)$, for $n>3$.

Lemma 3. The following equalities hold.

(i) $B_{n, \alpha, \beta}^{q}(1, x)=1$.

(ii) $B_{n, \alpha, \beta}^{q}(t, x)=\left([n+1]_{q}[n]_{q} / q^{2}[n-2]_{q}\left([n]_{q}+\beta\right)\right) x+$ $\left([n]_{q} / q[n-2]_{q}+\alpha\right)\left(1 /\left([n]_{q}+\beta\right)\right)$, for $n>2$.

(iii) $B_{n, \alpha, \beta}^{q}\left(t^{2}, x\right)=\left([n+1]_{q}[n+2]_{q}[n]_{q}^{2} / q^{6}[n-2]_{q}[n-\right.$ $\left.3]_{q}\left([n]_{q}+\beta\right)^{2}\right) x^{2}+\left([n]_{q}[2]_{q}^{2} / q^{3}[n-3]_{q}+2 \alpha\right)([n+$ $\left.1]_{q}[n]_{q} / q^{2}[n-2]\left([n]_{q}+\beta\right)^{2}\right) x+[2]_{q}[n]_{q}^{2} / q^{3}[n-2]_{q}[n-$ $3]_{q}\left([n]_{q}+\beta\right)^{2}+2 \alpha[n]_{q} / q[n-2]_{q}\left([n]_{q}+\beta\right)^{2}+\alpha^{2} /\left([n]_{q}+\right.$ $\beta)^{2}$, for $n>3$. 
Journal of Calculus of Variations

3

Proof. The operators $B_{n, \alpha, \beta}^{q}$ are well defined on function $1, t$, $t^{2}$. By Lemma 2 , for every $n>0$ and $x \in[0, \infty)$, we have

$$
\begin{aligned}
& B_{n, \alpha, \beta}^{q}(1, x)=B_{n}^{q}(1, x)=1 \\
B_{n, \alpha, \beta}^{q}(t, x)= & \frac{[n-1]_{q}}{[n]_{q}} \sum_{k=0}^{\infty} b_{n, k}^{q}(x) \\
& \times \int_{0}^{\infty / A} q^{k} p_{n, k}^{q}(t)\left(\frac{[n]_{q} t+\alpha}{[n]_{q}+\beta}\right) d_{q} t \\
= & \frac{[n]_{q}}{\left([n]_{q}+\beta\right)} B_{n}^{q}(t, x)+\frac{\alpha}{\left([n]_{q}+\beta\right)} B_{n}^{q}(t, x) \\
= & \frac{[n+1]_{q}[n]_{q}}{q^{2}[n-2]_{q}\left([n]_{q}+\beta\right)} x \\
& +\left(\frac{[n]_{q}}{q[n-2]_{q}}+\alpha\right) \frac{1}{\left([n]_{q}+\beta\right)} .
\end{aligned}
$$

Similarly,

$$
\begin{aligned}
B_{n, \alpha, \beta}^{q}\left(t^{2}, x\right)= & \frac{[n-1]_{q}}{[n]_{q}} \sum_{k=0}^{\infty} b_{n, k}^{q}(x) \\
& \times \int_{0}^{\infty / A} q^{k} p_{n, k}^{q}(t)\left(\frac{[n]_{q} t+\alpha}{[n]_{q}+\beta}\right)^{2} d_{q} t \\
= & \left.\frac{[n]_{q}}{[n]_{q}+\beta}\right)^{2} B_{n}^{q}\left(t^{2}, x\right) \\
& +\frac{2 \alpha[n]_{q}}{\left([n]_{q}+\beta\right)^{2}} B_{n}^{q}(t, x) \\
& +\left(\frac{\alpha}{[n]_{q}+\beta}\right)^{2} B_{n}^{q}(1, x) \\
& +\frac{[n+1]_{q}[n+2]_{q}[n]_{q}^{2}}{q^{6}[n-2]_{q}[n-3]_{q}\left([n]_{q}+\beta\right)^{2}} x^{2} \\
& +\left(\frac{[n]_{q}[2]_{q}^{2}}{q^{3}[n-3]_{q}}+2 \alpha\right) \\
& \times \frac{[n+1]_{q}[n]_{q}}{q^{2}[n-2]\left([n]_{q}+\beta\right)^{2}} x
\end{aligned}
$$

Remark 4. For all $m \in\{0,1,2, \ldots\}, 0 \leq \alpha \leq \beta$, we have the following recursive relation for the images of the monomials $t^{m}$ under $B_{n, \alpha, \beta}^{q}\left(t^{m}, x\right)$ in terms of $B_{n}^{q}\left(t^{r}, x\right), r=0,1,2, \ldots, m$, as

$$
B_{n, \alpha, \beta}^{q}\left(t^{m}, x\right)=\sum_{r=0}^{m}\left(\begin{array}{c}
m \\
r
\end{array}\right) \frac{n^{r} \alpha^{m-r}}{(n+\beta)^{m}} B_{n}^{q}\left(t^{r}, x\right) .
$$

Remark 5. If we put $q=1$ and $\alpha=\beta=0$, we get the moments of the modified Beta operators [6] as

$$
\begin{gathered}
B_{n}^{1}(t, x)=\frac{(n+1) x+1}{n-2}, \quad n>2, \\
B_{n}^{1}\left(t^{2}, x\right)=\frac{(n+1)(n+2) x^{2}+4(n+1) x+2}{(n-2)(n-3)}, \quad n>3 .
\end{gathered}
$$

Remark 6. From Lemma 3, we have$$
D_{n, \alpha, \beta}(x)=B_{n, \alpha, \beta}^{q}((t-x), x)
$$$$
=\left(\frac{[n+1]_{q}[n]_{q}}{q^{2}[n-2]_{q}\left([n]_{q}+\beta\right)}-1\right) x
$$$$
+\left(\frac{[n]_{q}}{q[n-2]_{q}}+\alpha\right) \frac{1}{\left([n]_{q}+\beta\right)}, \quad \text { for } n>2 \text {, }
$$$$
E_{n, \alpha, \beta}(x)=B_{n, \alpha, \beta}^{q}\left((t-x)^{2}, x\right)
$$$$
=B_{n, \alpha, \beta}^{q}\left(t^{2}, x\right)-2 x B_{n, \alpha, \beta}^{q}(t, x)+x^{2}
$$$$
=\left(\frac{[n+1]_{q}[n+2]_{q}[n]_{q}^{2}}{q^{6}[n-2]_{q}[n-3]_{q}\left([n]_{q}+\beta\right)^{2}}\right.
$$$$
\left.-\frac{2[n+1]_{q}[n]_{q}}{q^{2}[n-2]_{q}\left([n]_{q}+\beta\right)}+1\right) x^{2}
$$$$
+\left(\frac{[n+1]_{q}[n]_{q}^{2}[2]_{q}^{2}}{q^{5}[n-2]_{q}[n-3]_{q}\left([n]_{q}+\beta\right)^{2}}\right.
$$$$
+\frac{2[n+1]_{q}[n]_{q} \alpha}{q^{2}[n-2]_{q}\left([n]_{q}+\beta\right)^{2}}
$$$$
\left.-2\left(\frac{[n]_{q}}{q[n-2]_{q}}+\alpha\right) \frac{1}{\left([n]_{q}+\beta\right)}\right) x
$$$$
+\frac{[2]_{q}[n]_{q}^{2}}{q^{3}[n-2]_{q}[n-3]_{q}\left([n]_{q}+\beta\right)^{2}}
$$$$
+\frac{2 \alpha[n]_{q}}{q[n-2]_{q}\left([n]_{q}+\beta\right)^{2}}+\frac{\alpha^{2}}{\left([n]_{q}+\beta\right)^{2}},
$$

(19) 


\section{Direct Result and Asymptotic Formula}

Let the space $C_{B}[0, \infty)$ of all real-valued continuous bounded functions be endowed with the norm $\|f\|=\sup \{|f(t)|: x \in$ $[0, \infty)\}$. Further, let us consider the following $K$-functional:

$$
K_{2}(f ; \delta)=\inf _{g \in C^{2}[0, \infty)}\left\{\|f-g\|+\delta\left\|g^{\prime \prime}\right\|\right\},
$$

where $\delta>0$ and $C_{B}^{2}=\left\{g \in C_{B}[0, \infty): g^{\prime}, g^{\prime \prime} \in\right.$ $\left.C_{B}[0, \infty)\right\}$. By [15, page 177, Theorem 2.4], there exists an absolute constant $M>0$ such that

$$
K_{2}(f ; \delta) \leq M \omega_{2}(f ; \sqrt{\delta}),
$$

where

$$
\begin{aligned}
& \omega_{2}(f ; \sqrt{\delta}) \\
& =\sup _{0<h \leq \sqrt{\delta}}\left(\sup _{x \in[0, \infty)}|f(x+2 h)-2 f(x+h)+f(x)|\right)
\end{aligned}
$$

is the second-order modulus of smoothness of $f \in C_{B}[0, \infty)$. Also we set

$$
\omega(f ; \delta)=\sup _{0<h \leq \sqrt{\delta}}\left(\sup _{x \in[0, \infty)}|f(x+h)-f(x)|\right) .
$$

Theorem 7. Let $f \in C_{B}[0, \infty)$ and $q=q_{n} \in(0,1)$ such that $q_{n} \rightarrow 1$ as $n \rightarrow \infty$. Then for all $x \in[0, \infty)$ and $n>3$, there exists an absolute constant $C>0$ such that

$$
\begin{aligned}
\left|B_{n, \alpha, \beta}^{q}(f ; x)-f(x)\right| \leq & C \omega_{2}\left(f ; \sqrt{E_{n, \alpha, \beta}(x)+D_{n, \alpha, \beta}^{2}(x)}\right) \\
& +\omega\left(f ;\left|D_{n, \alpha, \beta}(x)\right|\right) .
\end{aligned}
$$

Proof. We are introducing the auxiliary operators as follows:

$$
\begin{aligned}
& \widehat{B}_{n, \alpha, \beta}^{q}(f ; x) \\
&=B_{n, \alpha, \beta}^{q}(f ; x)+f(x) \\
&-f\left(\frac{[n+1]_{q}[n]_{q}}{q^{2}[n-2]_{q}\left([n]_{q}+\beta\right)}\right. \\
&\left.\quad \times\left(x+\frac{q^{2} \alpha[n-2]_{q}}{[n]_{q}[n+1]_{q}}+\frac{q}{[n+1]_{q}}\right)\right) .
\end{aligned}
$$

From (25) and Lemma 3, we have

$$
\widehat{B}_{n, \alpha, \beta}^{q}(t-x ; x)=0 .
$$

Let $x \in[0, \infty)$ and $g \in C_{B}{ }^{2}[0, \infty)$. Using Taylor's formula

$$
g(t)-g(x)=(t-x) g^{\prime}(x)+\int_{x}^{t}(t-u) g^{\prime \prime}(u) d u,
$$

applying $\widehat{B}_{n, \alpha, \beta}^{q}$, and by (26), we get

$$
\begin{aligned}
& \widehat{B}_{n, \alpha, \beta}^{q}(g ; x)-g(x) \\
& =\widehat{B}_{n, \alpha, \beta}^{q}\left((t-x) g^{\prime}(x) ; x\right) \\
& +\widehat{B}_{n, \alpha, \beta}^{q}\left(\int_{x}^{t}(t-u) g^{\prime \prime}(u) d u ; x\right) \\
& =g^{\prime}(x) \widehat{B}_{n, \alpha, \beta}^{q}((t-x) ; x) \\
& +B_{n, \alpha, \beta}^{q}\left(\int_{x}^{t}(t-u) g^{\prime \prime}(u) d u ; x\right) \\
& -\int_{x}^{\left([n+1]_{q}[n]_{q} / q^{2}[n-2]_{q}\left([n]_{q}+\beta\right)\right) x+\left([n]_{q} / q[n-2]_{q}+\alpha\right)\left(1 /\left([n]_{q}+\beta\right)\right)} \\
& \left(\frac{[n+1]_{q}[n]_{q}}{q^{2}[n-2]_{q}\left([n]_{q}+\beta\right)} x\right. \\
& \left.+\left(\frac{[n]_{q}}{q[n-2]_{q}}+\alpha\right) \frac{1}{\left([n]_{q}+\beta\right)}-u\right) g^{\prime \prime}(u) d u \\
& =B_{n, \alpha, \beta}^{q}\left(\int_{x}^{t}(t-u) g^{\prime \prime}(u) d u ; x\right) \\
& -\int_{x}^{[n+1]_{q}[n]_{q} x / q^{2}[n-2]_{q}\left([n]_{q}+\beta\right)+\left([n]_{q} / q[n-2]_{q}+\alpha\right)\left(1 /\left([n]_{q}+\beta\right)\right)} \\
& \left(\frac{[n+1]_{q}[n]_{q} x}{q^{2}[n-2]_{q}\left([n]_{q}+\beta\right)}\right. \\
& \left.+\left(\frac{[n]_{q}}{q[n-2]_{q}}+\alpha\right) \frac{1}{\left([n]_{q}+\beta\right)}-u\right) g^{\prime \prime}(u) d u .
\end{aligned}
$$

On the other hand, since

$$
\begin{gathered}
\left|\int_{x}^{t}\right| t-u|| g^{\prime \prime}(u)|d u| \leq\left\|g^{\prime \prime}\right\|(t-x)^{2}, \\
\mid \int_{x}^{[n+1]_{q}[n]_{q} x / q^{2}[n-2]_{q}\left([n]_{q}+\beta\right)+\left([n]_{q} / q[n-2]_{q}+\alpha\right)\left(1 /\left([n]_{q}+\beta\right)\right)}
\end{gathered}
$$$$
\left(\frac{[n+1]_{q}[n]_{q} x}{q^{2}[n-2]_{q}\left([n]_{q}+\beta\right)}+\left(\frac{[n]_{q}}{q[n-2]_{q}}+\alpha\right)\right.
$$

$$
\left.\times \frac{1}{\left([n]_{q}+\beta\right)}-u\right) g^{\prime \prime}(u) d u
$$


Journal of Calculus of Variations

5

$$
\begin{aligned}
& \leq\left\|g^{\prime \prime}\right\|\left(\frac{[n+1]_{q}[n]_{q} x}{q^{2}[n-2]_{q}\left([n]_{q}+\beta\right)}\right. \\
& \left.\quad+\left(\frac{[n]_{q}}{q[n-2]_{q}}+\alpha\right) \frac{1}{\left([n]_{q}+\beta\right)}-x\right)^{2} \\
& =D_{n, \alpha, \beta}^{2}(x)\left\|g^{\prime \prime}\right\| .
\end{aligned}
$$

We conclude by Remark 6 that

$$
\begin{aligned}
& \left|\widehat{B}_{n, \alpha, \beta}^{q}(g ; x)-g(x)\right| \\
& =\left|B_{n, \alpha, \beta}^{q}\left(\int_{x}^{t}(t-u) g^{\prime \prime}(u) d u ; x\right)\right| \\
& +\mid \int_{x}^{[n+1]_{q}[n]_{q} x / q^{2}[n-2]_{q}\left([n]_{q}+\beta\right)+\left([n]_{q} / q[n-2]_{q}+\alpha\right)\left(1 /\left([n]_{q}+\beta\right)\right)} \\
& \left(\frac{[n+1]_{q}[n]_{q} x}{q^{2}[n-2]_{q}\left([n]_{q}+\beta\right)}\right. \\
& \left.+\left(\frac{[n]_{q}}{q[n-2]_{q}}+\alpha\right) \frac{1}{\left([n]_{q}+\beta\right)}-u\right) g^{\prime \prime}(u) d u \\
& \leq B_{n, \alpha, \beta}^{q}\left((t-x)^{2}\left\|g^{\prime \prime}\right\| ; x\right) \\
& +\left(\frac{[n+1]_{q}[n]_{q} x}{q^{2}[n-2]_{q}\left([n]_{q}+\beta\right)}\right. \\
& \left.+\left(\frac{[n]_{q}}{q[n-2]_{q}}+\alpha\right) \frac{1}{\left([n]_{q}+\beta\right)}-x\right)^{2}\left\|g^{\prime \prime}\right\|
\end{aligned}
$$

$$
\begin{gathered}
\left|\widehat{B}_{n, \alpha, \beta}^{q}(g ; x)-g(x)\right| \\
=E_{n, \alpha, \beta}(x)\left\|g^{\prime \prime}\right\| \\
\quad+D_{n, \alpha, \beta}^{2}(x)\left\|g^{\prime \prime}\right\| .
\end{gathered}
$$

From (25), we can write that

$$
\begin{aligned}
& \left|B_{n, \alpha, \beta}^{q}(f ; x)-f(x)\right| \\
& \leq\left|\widehat{B}_{n, \alpha, \beta}^{q}(f ; x)-f(x)\right| \\
& +\mid f(x)-f\left(\frac{[n+1]_{q}[n]_{q}}{q^{2}[n-2]_{q}\left([n]_{q}+\beta\right)}\right. \\
& \left.\times\left(x+\frac{q^{2} \alpha[n-2]_{q}}{[n]_{q}[n+1]_{q}}+\frac{q}{[n+1]_{q}}\right)\right)
\end{aligned}
$$

$$
\begin{aligned}
\leq\left|\widehat{B}_{n, \alpha, \beta}^{q}(f-g ; x)-(f-g)(x)\right|+\left|\widehat{B}_{n, \alpha, \beta}^{q}(g ; x)-g(x)\right| \\
+\mid f(x)-f\left(\frac{[n+1]_{q}[n]_{q}}{q^{2}[n-2]_{q}\left([n]_{q}+\beta\right)}\right. \\
\left.\quad \times\left(x+\frac{q^{2} \alpha[n-2]_{q}}{[n]_{q}[n+1]_{q}}+\frac{q}{[n+1]_{q}}\right)\right) \mid .
\end{aligned}
$$

Now, taking into account the boundedness of $B_{n, \alpha, \beta}^{q}$ and from (31), we get

$$
\begin{aligned}
& \left|B_{n, \alpha, \beta}^{q}(f ; x)-f(x)\right| \\
& \leq 4\|f-g\| \\
& +\left(E_{n, \alpha, \beta}(x)+D_{n, \alpha, \beta}^{2}(x)\right)\left\|g^{\prime \prime}\right\| \\
& +\mid f(x)-f\left(\frac{[n+1]_{q}[n]_{q}}{q^{2}[n-2]_{q}\left([n]_{q}+\beta\right)}\right. \\
& \left.\times\left(x+\frac{q^{2} \alpha[n-2]_{q}}{[n]_{q}[n+1]_{q}}+\frac{q}{[n+1]_{q}}\right)\right) \\
& \leq 4\|f-g\|+\omega\left(f ;\left|D_{n, \alpha, \beta}(x)\right|\right) \\
& +\left(E_{n, \alpha, \beta}(x)+D_{n, \alpha, \beta}^{2}(x)\right)\left\|g^{\prime \prime}\right\| .
\end{aligned}
$$

Now, taking infimum on the right-hand side over all $g \in$ $C_{B}{ }^{2}[0, \infty)$ and from (21), we get

$$
\begin{aligned}
\mid B_{n, \alpha, \beta}^{q}( & f ; x)-f(x) \mid \\
\leq & 4 K_{2}\left(f ; E_{n, \alpha, \beta}(x)+D_{n, \alpha, \beta}^{2}(x)\right) \\
& +\omega\left(f ;\left|D_{n, \alpha, \beta}(x)\right|\right) \\
= & C \omega_{2}\left(f ; \sqrt{E_{n, \alpha, \beta}(x)+D_{n, \alpha, \beta}^{2}(x)}\right) \\
& +\omega\left(f ;\left|D_{n, \alpha, \beta}(x)\right|\right),
\end{aligned}
$$

where $4 C_{1}=C>0$. This proves the theorem.

Our next result in this section is an asymptotic formula.

Theorem 8. Let $f$ be bounded and integrable function on the interval $[0, \infty)$; the second derivative of $f$ exists at a fixed point $x \in[0, \infty)$ and $q=q_{n} \in(0,1)$ such that $q_{n} \rightarrow 1$ as $n \rightarrow \infty$. Consider

$$
\begin{aligned}
\lim _{n \rightarrow \infty} & {[n]_{q_{n}}\left[B_{n, \alpha, \beta}^{q_{n}}(f ; x)-f(x)\right] } \\
\quad= & ((3-\beta) x+(1+\alpha)) f^{\prime}(x)+x(1+x) f^{\prime \prime}(x) .
\end{aligned}
$$


Proof. Using Taylor's expansion of $f$, we can write

$$
\begin{aligned}
f(t)-f(x)= & (t-x) f^{\prime}(x)+\frac{(t-x)^{2}}{2 !} f^{\prime \prime}(x) \\
& +\varepsilon(t-x)(t-x)^{2}
\end{aligned}
$$

where $\varepsilon$ is bounded and $\lim _{t \rightarrow 0} \varepsilon(t)=0$. Applying the operator $B_{n, \alpha, \beta}^{q_{n}}$ to the above relation, we get

$$
\begin{aligned}
B_{n, \alpha, \beta}^{q_{n}} & (f ; x)-f(x) \\
= & f^{\prime}(x) B_{n, \alpha, \beta}^{q_{n}}((t-x) ; x) \\
& +\frac{f^{\prime \prime}(x)}{2 !} B_{n, \alpha, \beta}^{q_{n}}\left((t-x)^{2} ; x\right) \\
& +B_{n, \alpha, \beta}^{q_{n}}\left(\varepsilon(t-x)(t-x)^{2} ; x\right) \\
= & f^{\prime}(x) D_{n, \alpha, \beta}(x)+\frac{f^{\prime \prime}(x)}{2 !} E_{n, \alpha, \beta}(x) \\
& +B_{n, \alpha, \beta}^{q_{n}}\left(\varepsilon(t-x)(t-x)^{2} ; x\right),
\end{aligned}
$$

where $D_{n, \alpha, \beta}(x)$ and $E_{n, \alpha, \beta}(x)$ are defined in Remark 6 .

Using Cauchy-Schwarz inequality, we have

$$
\begin{aligned}
& {[n]_{q_{n}} B_{n, \alpha, \beta}^{q_{n}}\left(\varepsilon(t-x)(t-x)^{2} ; x\right)} \\
& \quad \leq B_{n, \alpha, \beta}^{q_{n}}\left(\varepsilon^{2}(t-x) ; x\right)^{1 / 2}\left([n]_{q_{n}}^{2} B_{n, \alpha, \beta}^{q_{n}}\left((t-x)^{4} ; x\right)\right)^{1 / 2} .
\end{aligned}
$$

Using Theorem 1 with the help of Remark 4, we can easily find that

$$
\lim _{n \rightarrow \infty}[n]_{q_{n}}^{2} B_{n, \alpha, \beta}^{q_{n}}\left((t-x)^{4} ; x\right)=0 .
$$

Also, since

$$
\begin{gathered}
\lim _{n \rightarrow \infty}[n]_{q_{n}} D_{n, \alpha, \beta}(x)=(3-\beta) x+(1+\alpha), \\
\lim _{n \rightarrow \infty}[n]_{q_{n}} E_{n, \alpha, \beta}=2 x(1+x) .
\end{gathered}
$$

Thus,

$$
\begin{aligned}
\lim _{n \rightarrow \infty} & {[n]_{q_{n}}\left[B_{n, \alpha, \beta}^{q_{n}}(f ; x)-f(x)\right] } \\
& =((3-\beta) x+(1+\alpha)) f^{\prime}(x)+x(1+x) f^{\prime \prime}(x),
\end{aligned}
$$

which completes the proof.

\section{Better Estimation}

It is well known that the operators preserve constant as well as linear functions. To make the convergence faster, King [16] proposed an approach to modify the classical Bernstein polynomials, so that this sequence preserves two test functions: $e_{0}$ and $e_{2}$. After this, several researchers have studied that many approximating operators, $L$, possess these properties; that is, $L\left(e_{i} ; x\right)=e_{i}(x)$, where $e_{i}(x)=x^{i}(i=$ $0,1)$ or $x^{i}(i=0,2)$, for example, Bernstein, Baskakov, and Baskakov-Durrmeyer-Stancu operators (see [4, 5, 17-19]).

As the operators $B_{n, \alpha, \beta}^{q}$ introduced in (3) preserve only the constant functions, further modification of these operators is proposed to be made so that the modified operators preserve the constant as well as linear functions. For this purpose, the modification of $B_{n, \alpha, \beta}^{q}$ is as follows:

$$
\begin{aligned}
\widetilde{B}_{n, \alpha, \beta}^{q}(f ; x)= & B_{n, \alpha, \beta}^{q}\left(f ; r_{n, q}(x)\right) \\
= & \frac{[n-1]_{q}}{[n]_{q}} \sum_{k=0}^{\infty} b_{n, k}^{q}\left(r_{n, q}(x)\right) \\
& \times \int_{0}^{\infty / A} q^{k} p_{n, k}^{q}(t) f\left(\frac{[n]_{q} t+\alpha}{[n]_{q}+\beta}\right) d_{q} t,
\end{aligned}
$$

where

$$
\begin{aligned}
& r_{n, q}(x) \\
& =\left(\frac{-q[n]_{q}-\alpha q^{2}[n-2]_{q}+q^{2} x[n-2]_{q}\left([n]_{q}+\beta\right)}{[n]_{q}[n+1]_{q}}\right), \\
& x \in I_{n, q}=\left[\frac{[n]_{q}}{q[n-2]_{q}\left([n]_{q}+\beta\right)}+\frac{\alpha}{\left([n]_{q}+\beta\right)}, \infty\right) .
\end{aligned}
$$

Lemma 9. For each $x \in I_{n, q}$, one has

$$
\begin{aligned}
& \widetilde{B}_{n, \alpha, \beta}^{q}(1, x)=1, \quad \widetilde{B}_{n, \alpha, \beta}^{q}(t, x)=x, \\
& \widetilde{B}_{n, \alpha, \beta}^{q}\left(t^{2}, x\right)=\frac{[n-2]_{q}[n+2]_{q}}{q^{2}[n+1]_{q}[n-3]_{q}} x^{2}+\frac{1}{q^{3}[n-3]\left([n]_{q}+\beta\right)} \\
& \times\left([n]_{q}[2]_{q}^{2}+2 q^{3} \alpha[n-3]_{q}\right. \\
& \left.-\frac{2[n+2]_{q}\left([n]_{q}+q \alpha[n-2]_{q}\right)}{[n+1]_{q}}\right) x \\
& +\frac{1}{q^{4}[n-2]_{q}[n-3]_{q}\left([n]_{q}+\beta\right)^{2}} \\
& \times\left(\frac{[n+2]_{q}\left([n]_{q}+q \alpha[n-2]_{q}\right)^{2}}{[n+1]_{q}}\right. \\
& -[n]_{q}^{2}[2]_{q}-q \alpha[2]_{q}^{2}[n]_{q}[n-2]_{q} \\
& \left.-q^{4} \alpha^{2}[n-3]_{q}[n-2]_{q}\right), \quad \text { for } n>3 .
\end{aligned}
$$


Lemma 10. For each $x \in I_{n, q}$, the following equalities hold:

$$
\begin{aligned}
& \widetilde{B}_{n, \alpha, \beta}^{q}((t-x), x)=0, \\
& F_{n, \alpha, \beta}=\widetilde{B}_{n, \alpha, \beta}^{q}\left((t-x)^{2}, x\right) \\
& =\left(\frac{[n-2]_{q}[n+2]_{q}}{q^{2}[n+1]_{q}[n-3]_{q}}-1\right) x^{2} \\
& +\frac{1}{q^{3}[n-3]\left([n]_{q}+\beta\right)} \\
& \times\left([n]_{q}[2]_{q}^{2}-\frac{2[n+2]_{q}\left([n]_{q}+q \alpha[n-2]_{q}\right)}{[n+1]_{q}}\right. \\
& \left.+2 q^{3} \alpha[n-3]_{q}\right) x \\
& +\frac{1}{q^{4}[n-2]_{q}[n-3]_{q}\left([n]_{q}+\beta\right)^{2}} \\
& \times\left(\frac{[n+2]_{q}\left([n]_{q}+q \alpha[n-2]_{q}\right)^{2}}{[n+1]_{q}}\right. \\
& -[n]_{q}^{2}[2]_{q}-q \alpha[2]_{q}^{2}[n]_{q}[n-2]_{q} \\
& \left.-q^{4} \alpha^{2}[n-3]_{q}[n-2]_{q}\right), \quad \text { for } n>3 .
\end{aligned}
$$

Theorem 11. Let $f \in C_{B}\left(I_{n, q}\right)$ and $q=q_{n} \in(0,1)$ such that $q_{n} \rightarrow 1$ as $n \rightarrow \infty$. Then for all $x \in I_{n, q}$ and $n>3$, there exists an absolute constant $C>0$ such that

$$
\left|\widetilde{B}_{n, \alpha, \beta}^{q}(f ; x)-f(x)\right| \leq C \omega_{2}\left(f ; \sqrt{F_{n, \alpha, \beta}(x)}\right) .
$$

Proof. Let $x, t \in I_{n, q}$ and $g \in C_{B}^{2}\left(I_{n, q}\right)$. Using Taylor's formula, we get

$$
g(t)-g(x)=(t-x) g^{\prime}(x)+\int_{x}^{t}(t-u) g^{\prime \prime}(u) d u \text {. }
$$

Applying $\widetilde{B}_{n, \alpha, \beta}^{q}$, we get

$$
\begin{aligned}
\widetilde{B}_{n, \alpha, \beta}^{q}(g ; x)-g(x) \\
=g^{\prime}(x) \widetilde{B}_{n, \alpha, \beta}^{q}((t-x) ; x) \\
\quad+\widetilde{B}_{n, \alpha, \beta}^{q}\left(\int_{x}^{t}(t-u) g^{\prime \prime}(u) d u ; x\right) .
\end{aligned}
$$

Obviously, we have

$$
\left|\int_{x}^{t}\right| t-u|| g^{\prime \prime}(u)|d u| \leq\left\|g^{\prime \prime}\right\|(t-x)^{2} .
$$

Therefore,

$$
\begin{aligned}
\left|\widetilde{B}_{n, \alpha, \beta}^{q}(g ; x)-g(x)\right| & \leq\left\|g^{\prime \prime}\right\| \widetilde{B}_{n, \alpha, \beta}^{q}\left((t-x)^{2} ; x\right) \\
& =F_{n, \alpha, \beta}\left\|g^{\prime \prime}\right\| .
\end{aligned}
$$

Since $\left|\widetilde{B}_{n, \alpha, \beta}^{q}(f ; x)-f(x)\right| \leq 2\|f\|$, thus

$$
\begin{aligned}
\left|\widetilde{B}_{n, \alpha, \beta}^{q}(f ; x)-f(x)\right| \\
\leq\left|\widetilde{B}_{n, \alpha, \beta}^{q}(f-g ; x)-(f-g)(x)\right| \\
\quad+\left|\widetilde{B}_{n, \alpha, \beta}^{q}(g ; x)-g(x)\right| \\
\leq 2\|f-g\|+F_{n, \alpha, \beta}(x)\left\|g^{\prime \prime}\right\| .
\end{aligned}
$$

Now, taking infimum on the right-hand side over all $g \in$ $C_{B}^{2}\left(I_{n, q}\right)$ and from (21), we get

$$
\left|\widetilde{B}_{n, \alpha, \beta}^{q}(f ; x)-f(x)\right| \leq C \omega_{2}\left(f ; \sqrt{F_{n, \alpha, \beta}(x)}\right) .
$$

which proves the theorem.

Theorem 12. Let $f$ be bounded and integrable function on the interval $I_{n, q}$; the second derivative of $f$ exists at a fixed point $x \in I_{n, q}$ and $q=q_{n} \in(0,1)$ such that $q_{n} \rightarrow 1$ as $n \rightarrow \infty$; then

$$
\lim _{n \rightarrow \infty}[n]_{q_{n}}\left[\widetilde{B}_{n, \alpha, \beta}^{q_{n}}(f ; x)-f(x)\right]=x(1+x) f^{\prime \prime}(x) .
$$

The proof follows along the same lines of Theorem 8 .

\section{Conflict of Interests}

The authors declare that there is no conflict of interests.

\section{Acknowledgments}

The authors thank the anonymous learned reviewers for their valuable suggestions, which substantially improved the standard of the paper. Special thanks are due to Professor Adam Kowalewski, for kind cooperation and smooth behavior during communication and for his efforts to send the reports of the paper timely.

\section{References}

[1] N. K. Govil and V. Gupta, "Convergence of $q$ MeyerKonigZellerDurrmeyer operators," Advanced Studies in Contemporary Mathematics, vol. 19, pp. 97-108, 2009.

[2] V. Gupta, "A note on modified Baskakov type operators," Approximation Theory and Its Applications, vol. 10, no. 3, pp. 7478, 1994.

[3] V. Gupta, "Some approximation properties of $q$-Durrmeyer operators," Applied Mathematics and Computation, vol. 197, no. 1, pp. 172-178, 2008.

[4] A. Aral and V. Gupta, "Generalized q-Baskakov operators," Mathematica Slovaca, vol. 61, no. 4, pp. 619-634, 2011. 
[5] Z. Finta and V. Gupta, "Approximation properties of $q$-Baskakov operators," Central European Journal of Mathematics, vol. 8, no. 1, pp. 199-211, 2010.

[6] V. Gupta and A. Ahmad, "Simultaneous approximation by modified Beta operators," Istanbul Üniversitesi Fen Fakültesi Matematik Dergisi, vol. 54, pp. 11-22, 1996.

[7] P. Maheshwari and D. Sharma, "Approximation by $q$ BaskakovBeta-Stancu operators," Rendiconti del Circolo Matematico di Palermo, vol. 61, no. 2, pp. 297-305, 2012.

[8] V. Gupta and T. Kim, "On the rate of approximation by $q$ modified Beta operators," Journal of Mathematical Analysis and Applications, vol. 377, no. 2, pp. 471-480, 2011.

[9] V. Gupta, D. K. Verma, and P. N. Agrawal, "Simultaneous approximation by certain Baskakov-Durrmeyer-Stancu operators," Journal of the Egyptian Mathematical Society, vol. 20, no. 3, pp. 183-187, 2012.

[10] G. Gasper and M. Rahman, Basic Hypergeometric Series, vol. 35 of Encyclopedia of Applied and Computational Mathematics, Cambridge University Press, Cambridge, UK, 1990.

[11] V. G. Kac and P. Cheung, Quantum Calculus, Universitext, Springer, New York, NY, USA, 2002.

[12] T. Kim, " $q$-generalized Euler numbers and polynomials," Russian Journal of Mathematical Physics, vol. 13, no. 3, pp. 293-298, 2006.

[13] T. Kim, "Note on the Euler q-zeta functions," Journal of Number Theory, vol. 129, no. 7, pp. 1798-1804, 2009.

[14] A. De Sole and V. G. Kac, "On integral representation of $q$ gamma and $q$-beta functions," Atti-Accademia Nazionale Dei Lincei Rendiconti Lincei Classe Di Scienze Fisiche Matemaiche E Naturali Serie 9 Matematica E Applicazioni, vol. 1, no. 16, pp. 11-29, 2005.

[15] R. A. DeVore and G. G. Lorentz, Constructive Approximation, Springer, Berlin, Germany, 1993.

[16] J. P. King, "Positive linear operators which preserve $x_{2}$," Acta Mathematica Hungarica, vol. 99, no. 3, pp. 203-208, 2003.

[17] O. Agratini, "Linear operators that preserve some test functions," International Journal of Mathematics and Mathematical Sciences, Article ID 94136, 11 pages, 2006.

[18] O. Agratini, "On the iterates of a class of summation-type linear positive operators," Computers and Mathematics with Applications, vol. 55, no. 6, pp. 1178-1180, 2008.

[19] D. K. Verma, V. Gupta, and P. N. Agrawal, "Some approximation properties of Baskakov-Durrmeyer-Stancu operators," Applied Mathematics and Computation, vol. 218, no. 11, pp. 6549-6556, 2012. 


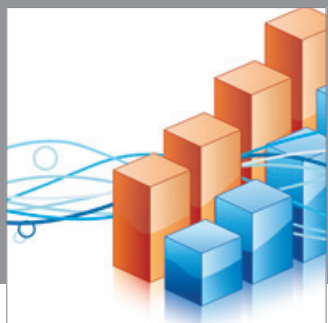

Advances in

Operations Research

mansans

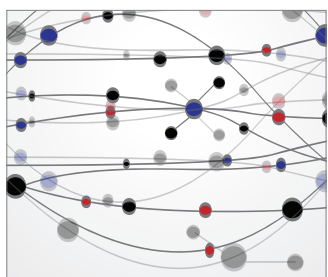

The Scientific World Journal
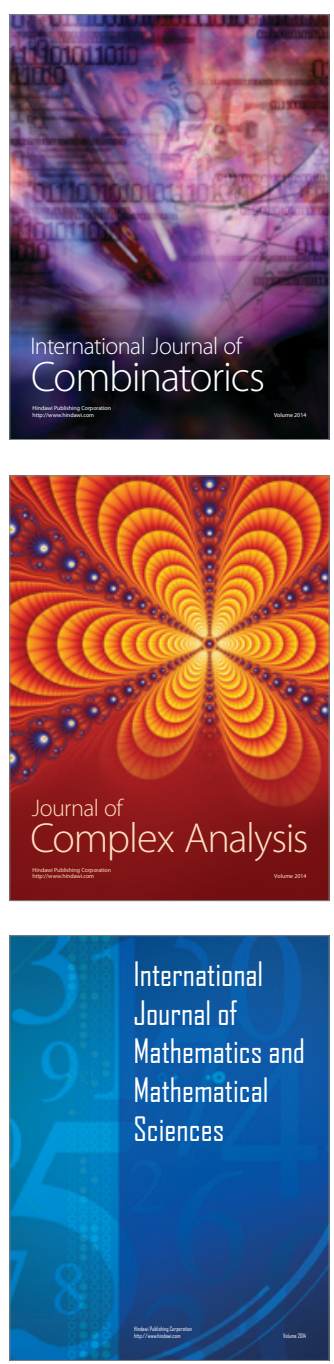
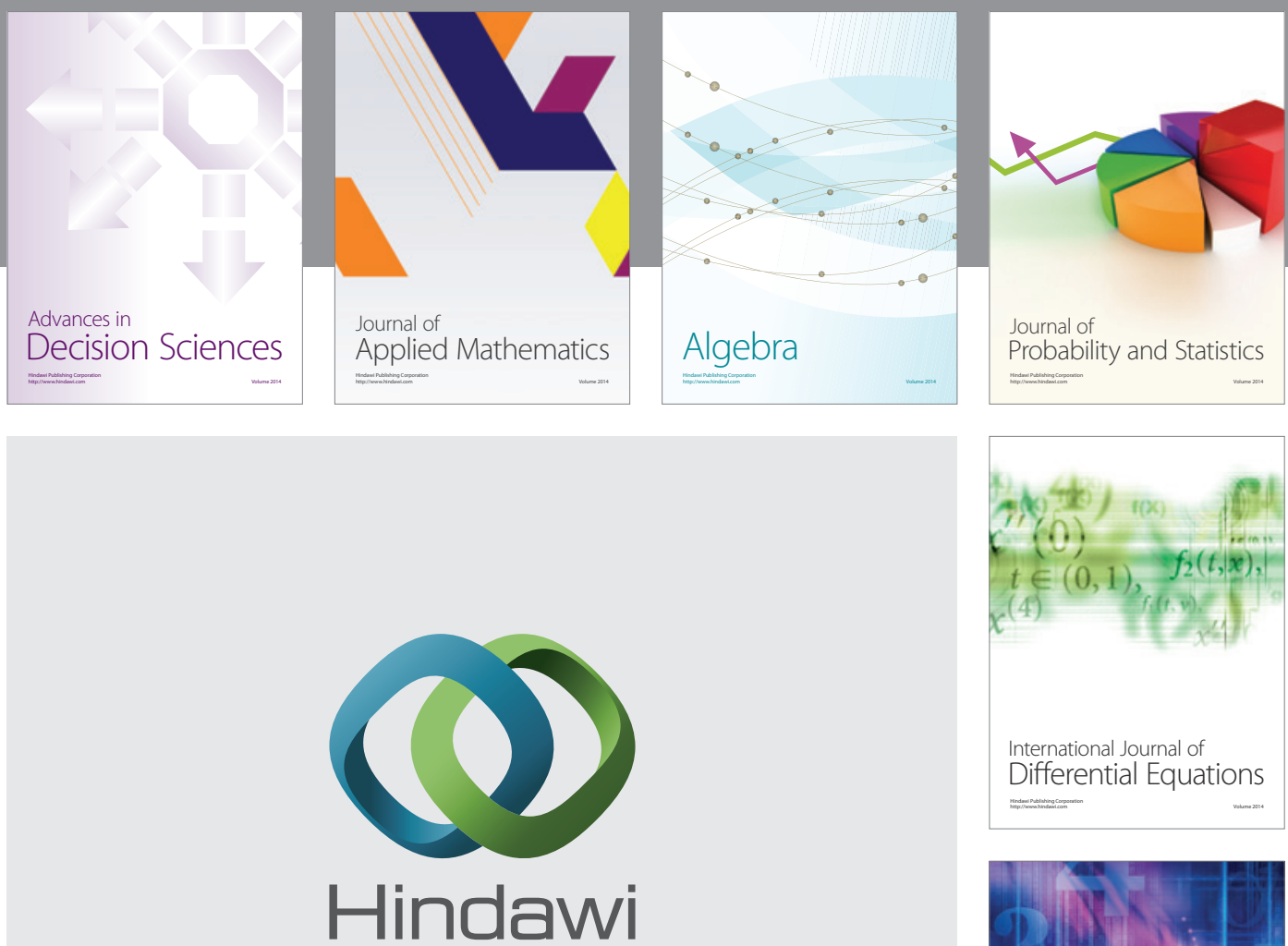

Submit your manuscripts at http://www.hindawi.com
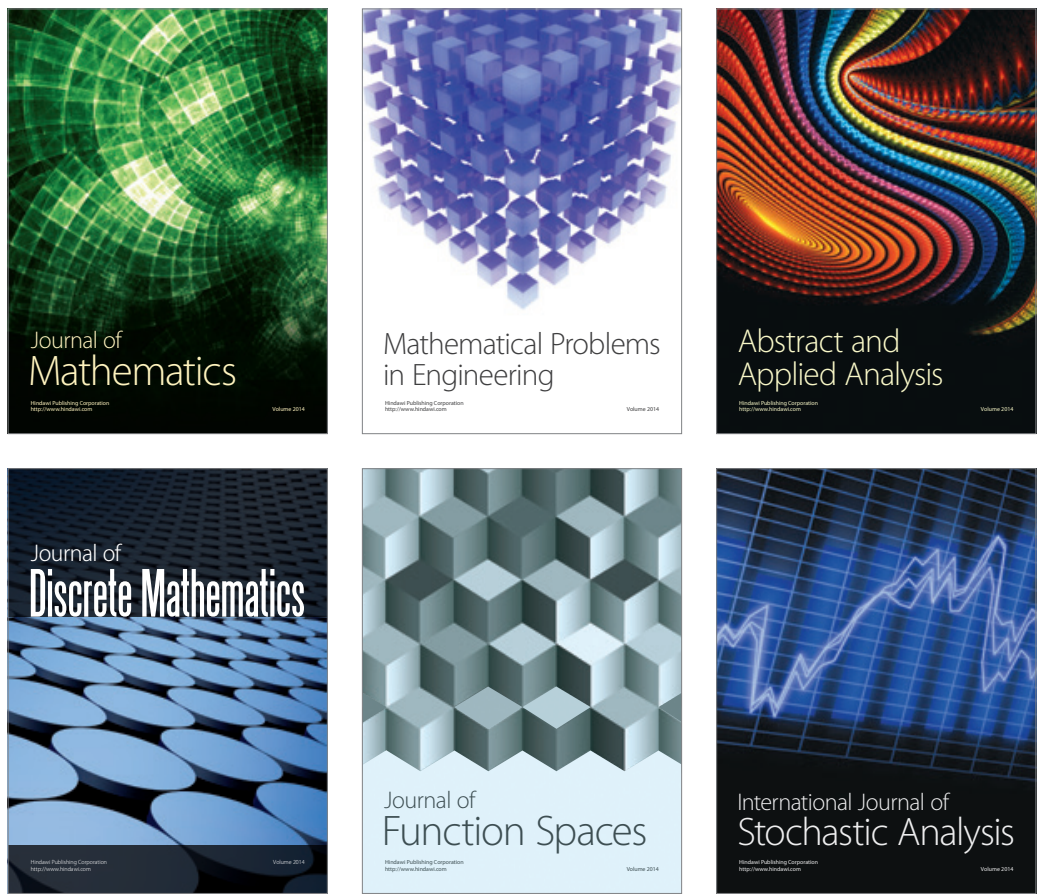

Journal of

Function Spaces

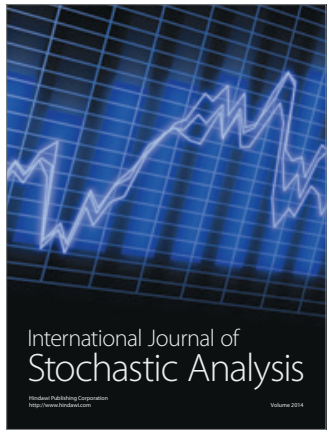

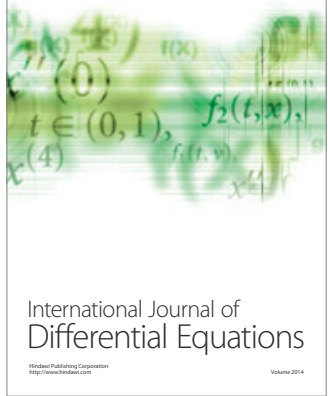
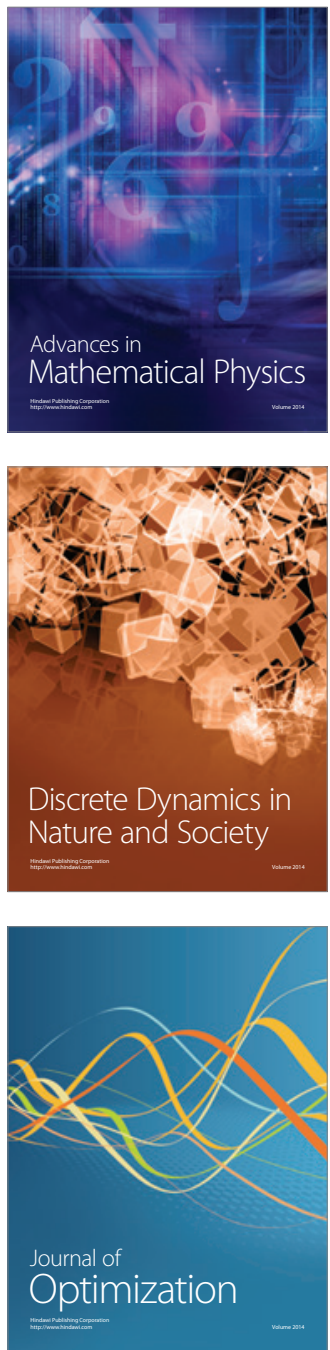